\title{
A Case of Acute Cauda Equina Syndrome for Combined Lumbar Ossification of the Posterior Longitudinal and Yellow Ligament
}

\author{
Kazumasa Nakamura, Yuichirou Yokoyama, Akihito Wada, Yasuhiro Inoue, \\ Keiji Hasegawa, Shintaro Tsuge, Hiroshi Takahashi*, Yasuaki Iida \\ Department of Orthopaedic Surgery, School of Medicine, Toho University, Tokyo, Japan \\ Email: ${ }^{*}$ drkan@med.toho-u.ac.jp
}

Received 17 April 2014; revised 28 May 2014; accepted 15 June 2014

Copyright (C) 2014 by authors and Scientific Research Publishing Inc.

This work is licensed under the Creative Commons Attribution International License (CC BY).

http://creativecommons.org/licenses/by/4.0/

\begin{abstract}
Acute cauda equina syndrome is known as a symptom of lumbar disc herniation, but to date, there have been no reports of cases caused by lumbar vertebral ligament ossification. We encountered a 61-year-old female patient with acute cauda equina syndrome associated with lumbar vertebral OPLL and OLF. The symptoms were improved by emergency laminectomy. One year after the surgery, the disturbances of gait and urination have been resolved.
\end{abstract}

\section{Keywords}

Lumbar Spine, Ossification of the Yellow Ligament, Ossification of the Posterior Longitudinal Ligament, Cauda Equina Syndrome

\section{Introduction}

In cases of ossification of the posterior longitudinal ligament (OPLL), lumbar cases occur less frequently than cervical or thoracic cases, and it is considered that neurologic manifestations of lumbar OPLL do not occur readily, due to specific anatomical characteristics. We encountered a rare case of acute cauda equina syndrome (CES) associated with OPLL and ossification of the yellow ligament (OYL) in the lumbar region.

\section{Case Presentation: A 61-Year-Old Woman}

Chief complaints: Numbness of the bilateral lower limbs and dysuria.

*Corresponding author.

How to cite this paper: Nakamura, K., Yokoyama, Y., Wada, A., Inoue, Y., Hasegawa, K., Tsuge, S., Takahashi, H. and lida, Y. (2014) A Case of Acute Cauda Equina Syndrome for Combined Lumbar Ossification of the Posterior Longitudinal and Yellow Ligament. Open Journal of Orthopedics, 4, 145-149. http://dx.doi.org/10.4236/ojo.2014.46024 
History of present illness: Right lower limb pain developed without apparent cause. It gradually progressed to numbness of the bilateral lower limbs and burning sensation of the perineal region and resulted in dysbasia. The patient then visited a physician.

She was treated with drip infusion of adrenocortical steroid, but the symptoms did not improve, and urinary retention developed. Thus, she was referred to our hospital on the next day. Acute cauda equina syndrome was suspected, and the patient was urgently admitted for surgery.

Past medical history: Hypertension and dyslipidemia

Status on admission: Height: $153 \mathrm{~cm}$; body weight: $93 \mathrm{~kg}$; and BMI: 40; showing marked obesity. Straight leg raising and femoral nerve stretch tests were negative bilaterally, and MMT was reduced (right EHL and FHL: 4). Regarding sensation, hypaesthesia was noted in the regions innervated by the left L5 nerve root and in the perineal area. Both urination and defecation were disturbed, and $600 \mathrm{ml}$ of residual urine was drained upon urine drainage.

Blood test: HbA1c, 5.6\%; insulin, $32.4 \mu \mathrm{IU} / \mathrm{ml}$; C-peptide, 4.99 ng/ml; GH, 0.14 ng/ml; IGF-1 50 ng/ml.

Physiological function test: R-ABI, 1.11; L-ABI, 1.07; R-TBI, 0.44; L-TBI, 0.56.

Imaging findings: On plain X-ray radiography of the lumbar spine, OPLL (white arrows) and OYL (black arrows) were noted at the L1/2, 2/3, 3/4, and 4/5 intervertebral disc levels. Ossification of the anterior longitudinal ligament (OALL) was also noted at the Th12/L1, L1/2, 2/3, 3/4, and 4/5 intervertebral levels (Figure 1).

Plain CT of the lumbar spine demonstrated mixed-type OPLL from the L1 to L5 level. In addition, OYL accompanied by OALL was noted at each intervertebral level. The spinal canal was severely narrowed by OPLL and OYL at the L3/4 and 4/5 intervertebral levels (Figure 2).

MRI of the lumbar spine demonstrated ossified lesions showing a low intensity on T1- and T2-weight imaging on the posterior surface of the L1-L5 vertebrae. These lesions excluded the dural canal and caused severe spinal canal stenosis at the L3/4 and 4/5 intervertebral levels (Figure 3).

Based on the above findings, the patient was diagnosed with acute cauda equina syndrome caused by lumbar OPLL and OYL, and emergency surgery was performed on the same day.

Surgical findings: L3, 4, and 5 laminectomy was performed. The stenosis at L3/4 and L4/5 was severe, OYL at L4/5 adhered to the dura mater, and the dura mater was partially ossified. The OYL at the L2/3, 3/4, 4/5, 5/S intervertebral levels were excised, and sufficient decompression was achieved from the L2/3 to L5/S region.

On postoperative imaging, the dural canal compression at the L2/3, 3/4, and 4/5 levels improved, showing favorable decompression (Figure 4).

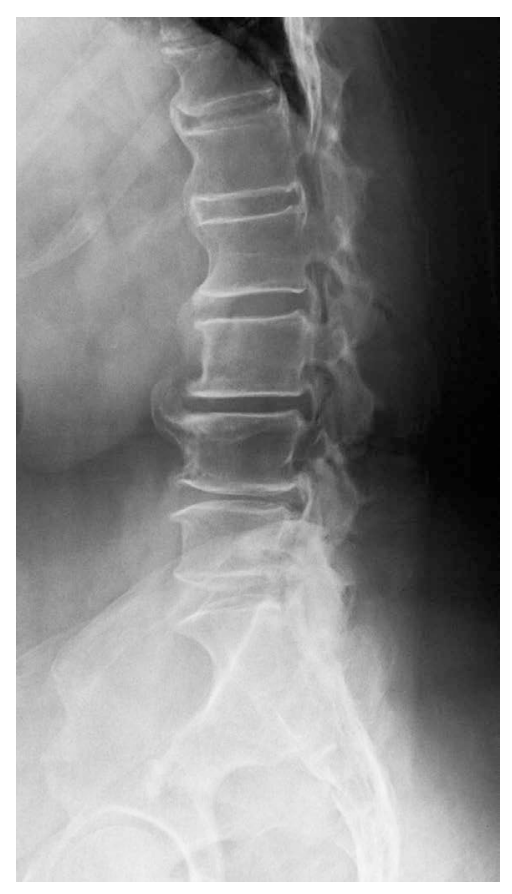

Figure 1. Plain X-ray radiography. OPLL, OLF, and OALL were observed in the lumbar vertebral region. 


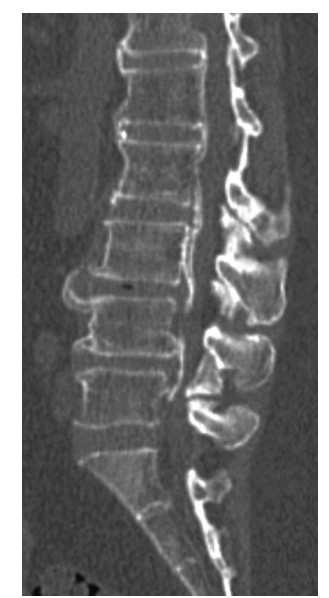

(a)

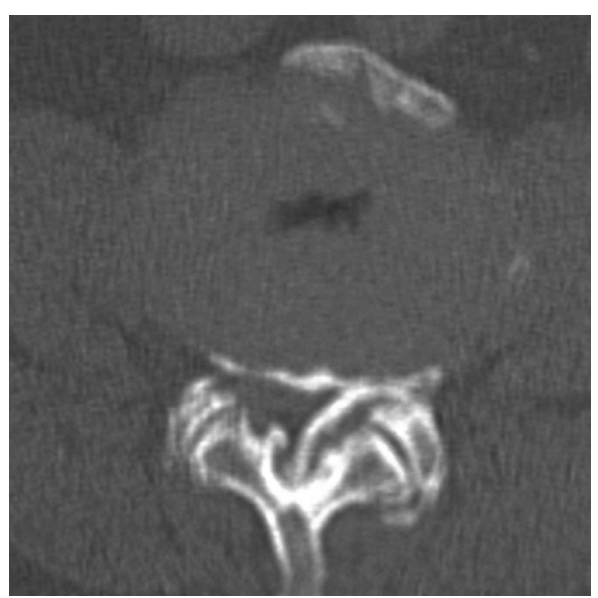

(b)

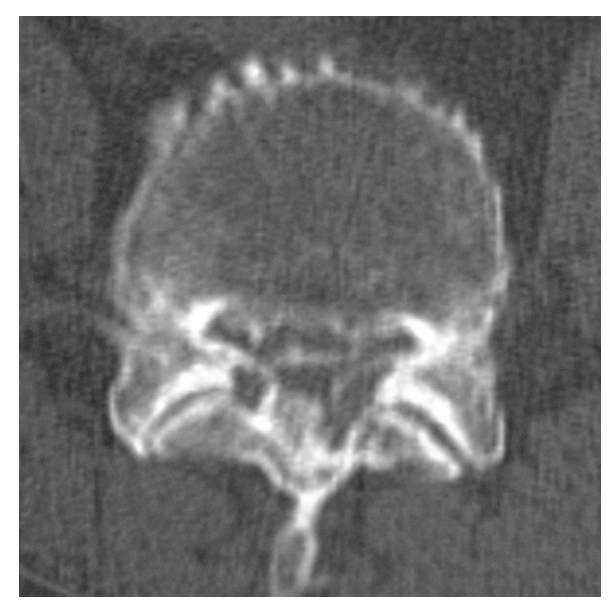

(c)

Figure 2. Plain CT on admission. (a) CT-MPR; (b) L3/4 level; (c) L4/5 level. Severe spinal canal stenosis was caused by OPLL and OLF at the L3/4 and L4/5 levels.

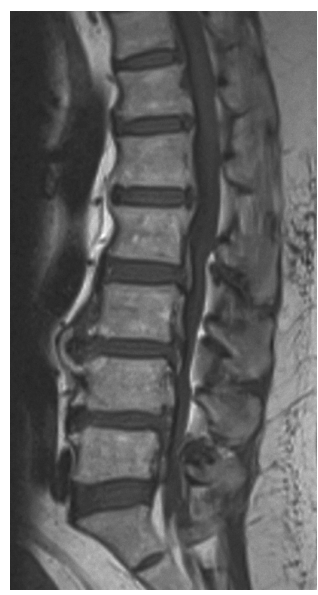

(a)

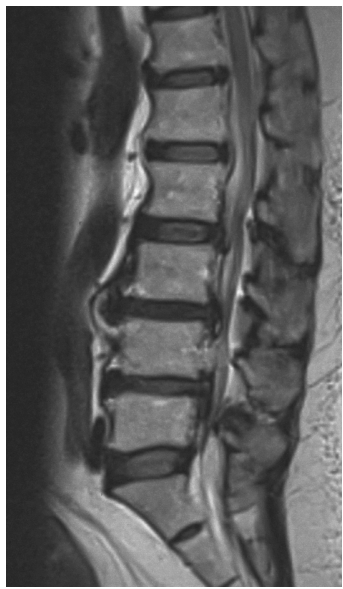

(b)

Figure 3. MRI on admission. (a) Sagittal T1WI; (b) Sagittal T2WI. Severe spinal canal stenosis caused by OPLL and OLF was noted.

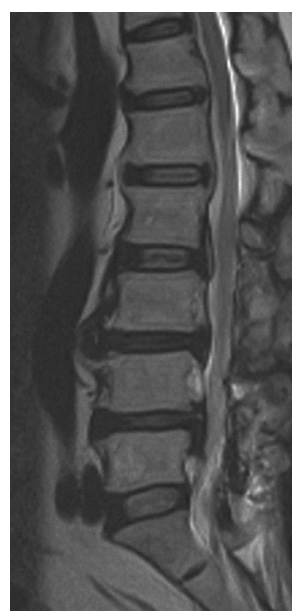

Figure 4. Postoperative MRI. On sagittal T2WI, the dural canal was favorably decompressed. 
Postoperative course: The numbness of the perineal region and lower limb improved on the day after the surgery. At one week after the surgery, the muscle weakness of the lower limbs was improved and patient became able to walk. At 2 weeks, she was able to urinate and defecate. On the final follow-up at 12 months after surgery, there was no gait disturbance, and only mild hypaesthesia of the perineal region and numbness around the bilateral ankle joints were noted.

\section{Discussion}

In general, the incidence of OPLL is lower in the lumbar than in the cervical spine.

Since there is sufficient room in the spinal canal and intervertebral foramen in the lumbar vertebral region, neurologic symptoms do not readily manifest, even when the posterior longitudinal ligament is ossified in the spinal canal, and severe stenosis must occur for the development of symptoms from the lower lumbar region compared with the upper lumbar region [1].

As generally represented by lumbar disc herniation, CES develops when a space-occupying lesion appears rapidly in the spinal canal, and the symptoms include severe lumbago, sciatica, saddle anesthesia, urinary incontinence, and muscle weakness and sensory disturbance of the lower limbs [2] [3].

OPLL/OYL-induced spinal canal stenosis is considered to progress slowly, and it is unlikely that CES is caused by OPLL- or OYL-induced spinal canal stenosis alone.

To our knowledge, there has been no reported case of OPLL/OYL-induced CES in Japan. We considered the developmental mechanism of lumbar vertebral OPLL/OYL-induced CES in this patient as follows:

Severe spinal canal stenosis was caused by OPLL and OYL was noted at the L3/4 and 4/5 levels in this patient, suggesting that the CES arose from these levels. Accordingly, it was assumed that another factor, in addition to severe stenosis, was involved in the development of CES. We considered that, in this patient, instability and peripheral arterial occlusive disease (PAD) in discontinuous intervertebral regions associated with diffuse idiopathic skeletal hyperostosis (DISH) were involved in the pathological development of the symptoms.

DISH was first reported as ankylosing spinal hyperostosis by Forestier et al. [4], and subsequently described as DISH by Resnick et al. [5]. The incidence is high in men aged 50 years or older, with normal intervertebral discs, and the disease is not typically accompanied by ossification of the sacroiliac joint and joints of the four limbs. Risk factors for DISH include type 2 diabetes [6]-[8], hyperuricemia [6]-[8], dyslipidemia [6], and high BMI [9], and it has been reported that serum insulin [9]-[11], GH [9], and IGF-1 levels [9] are significantly higher in DISH patients. These risk factors were mostly present in this patient.

Discontinuous and fragile regions of ossified spinal lesions have occasionally been reported to lead to spinal cord injury and neuropathy [12]-[15]. In our patient, OALL continuous from Th12 was discontinuous at L2/3, L3/4, and 4/5, and OPLL was also discontinuous at the L3/4 level. In addition, a vacuum phenomenon was noted in the L3/4 intervertebral disc, suggesting that intervertebral instability was present in these regions due to discontinuous DISH and OPLL and was involved in the development of CES.

Moreover, TBI was reduced on both sides in this patient, despite a normal ABI, suggesting the presence of peripheral arterial occlusive lesions [16]. Although the influence of peripheral arterial occlusive lesions on the circulatory dynamics in the cauda equine nerve can only be assumed, resistance of the cauda equine nerve to compression may have been weak due to reduced blood flow in the nerve.

It was suggested that OPLL- and OYL-induced severe stenosis of the spinal canal was combined with intervertebral instability and peripheral arterial occlusive lesions and caused CES, which occurs rarely due to lumbar vertebral ligament ossification.

A further study is expected about causation of ligament ossification and hyperinsulinemia in future.

\section{References}

[1] Noda, M. (1996) Clinical Study of the Ossification of Posterior Longitudinal Ligament of the Lumbar Spine. Yamaguchi Medical Journal, 45, 59-70. (in Japanese)

[2] Ahn, U.M., Ahn, N.U., Buchowski, J.M., Garrett, E.S., Sieber, A.N. and Kostuik, J.P. (2000) Cauda Equina Syndrome Secondary to Lumbar Disc Herniation A Meta-Analysis of Surgical Outcomes. Spine, 25, 1515-1522. http://dx.doi.org/10.1097/00007632-200006150-00010

[3] Kostuik, J.P., Harrington, I., Alexander, D., Rand, W. and Evans, D. (1986) Cauda Equina Syndrome and Lumbar Disc Herniation. The Journal of Bone and Joint Surgery, 68A, 386-391. 
[4] Forestier, J. and Rotes-Querol, J. (1950) Senile Ankylosing Hyperostosis of the Spine. Annals of the Rheumatic Diseases, 9, 321-330. http://dx.doi.org/10.1136/ard.9.4.321

[5] Resnick, D. and Niwayama, G. (1976) Radiographic and Pathologic Features of Spinal Involvement in Diffuse Idiopathic Skeletal Hyperostosis (DISH). Radiology, 119, 559-568.

[6] Vezyroglou, G., Mitropoulos, A. and Antoniadis, C. (1996) A Metabolic Syndrome in Diffuse Idiopathic Skeletal Hyperostosis: A Controlled Study. The Journal of Rheumatology, 23, 672-676.

[7] Kiss, C., Szilagyi, M., Paksy, A. and Poor, G. (2002) Risk Factor for Diffuse Idiopathic Skeletal Hyperostosis: A CaseControlled Study. Ruematology (Oxford), 41, 27-30. http://dx.doi.org/10.1093/rheumatology/41.1.27

[8] Sarzi-Puttini, P. and Atzeni, F. (2004) New Developments in Our Understanding of DISH (Diffuse Idiopathic Skeletal Hyperostosis). Current Opinion in Rheumatology, 16, 287-292. http://dx.doi.org/10.1097/00002281-200405000-00021

[9] Denko, C.W. and Malemud, C.J. (2006) Body Mass Index and Blood Glucose: Correlations with Serum Insulin, Growth Hormone, and Insulin-Like Growth Factor-1 Levels in Patients with Diffuse Idiopathic Skeletal Hyperostosis (DISH). Rheumatology International, 26, 292-297. http://dx.doi.org/10.1007/s00296-005-0588-8

[10] Denko, C.W., Boja, B. and Moskowitz, R.W. (1994) Growth Promoting Peptides in Osteoarthritis and Diffuse Idiopathic Skeletal Hyperostosis-Insulin, Insulin-Like Growth Factor-1, Growth Hormone. The Journal of Rheumatology, 21, 1725-1730.

[11] Littlejohn, G.O. and Smythe, H.A. (1981) Marked Hyperinsulinemia after Glucose Challenge in Patients with Diffuse Idiopathic Skeletal Hyperostosis. The Journal of Rheumatology, 8, 965-968.

[12] Houk, R.W., Hendrix, R.W., Lee, C., Lal, S. and Schmid, F.R. (1984) Cervical Fracture and Paraplegia Complicating Diffuse Idiopathic Skeletal Hyperostosis. Arthritis \& Rheumatism, 27, 472-475. http://dx.doi.org/10.1002/art.1780270418

[13] Yasumura, Y., Suetsuna, F., Todate, K., Ise, N. and Handa, A. (1996) Spinal Fracture Complicating Ankylosing Spinal Hyperostosis-Two Cases Report. Clinical Rheumatology, 8, 200-207. (in Japanese)

[14] Mehara, H., Sakaguchi, Y. and Ogura, H. (2002) Chance Fracture of the Thoracic Spine with Delayed Paraplegia in a Patient with Ankylosing Spinal Hyperostosis: A Case Report. Seikeigeka, 53, 1543-1546. (in Japanese)

[15] Yamada, H., Hirabayashi, S., Motosuneya, T., Kojima, J., Kobayashi, Y. and Sakai, H. (2007) Thoracic Spinal Instability at the Discontinuous Ossification of the Anterior Longitudinal Ligament in Patients with Diffuse Idiopathic Skeletal Hyperostosis: Report of Two Cases. Seikeigeka, 58, 525-529. (in Japanese)

[16] Shigematsu, H., et al. (2009) Guidelines for Management of Peripheral Arterial Occlusive Diseases (JCS 2009). Circulation Journal, 73, 1507-1558. 
Scientific Research Publishing (SCIRP) is one of the largest Open Access journal publishers. It is currently publishing more than 200 open access, online, peer-reviewed journals covering a wide range of academic disciplines. SCIRP serves the worldwide academic communities and contributes to the progress and application of science with its publication.

Other selected journals from SCIRP are listed as below. Submit your manuscript to us via either submit@scirp.org or Online Submission Portal.
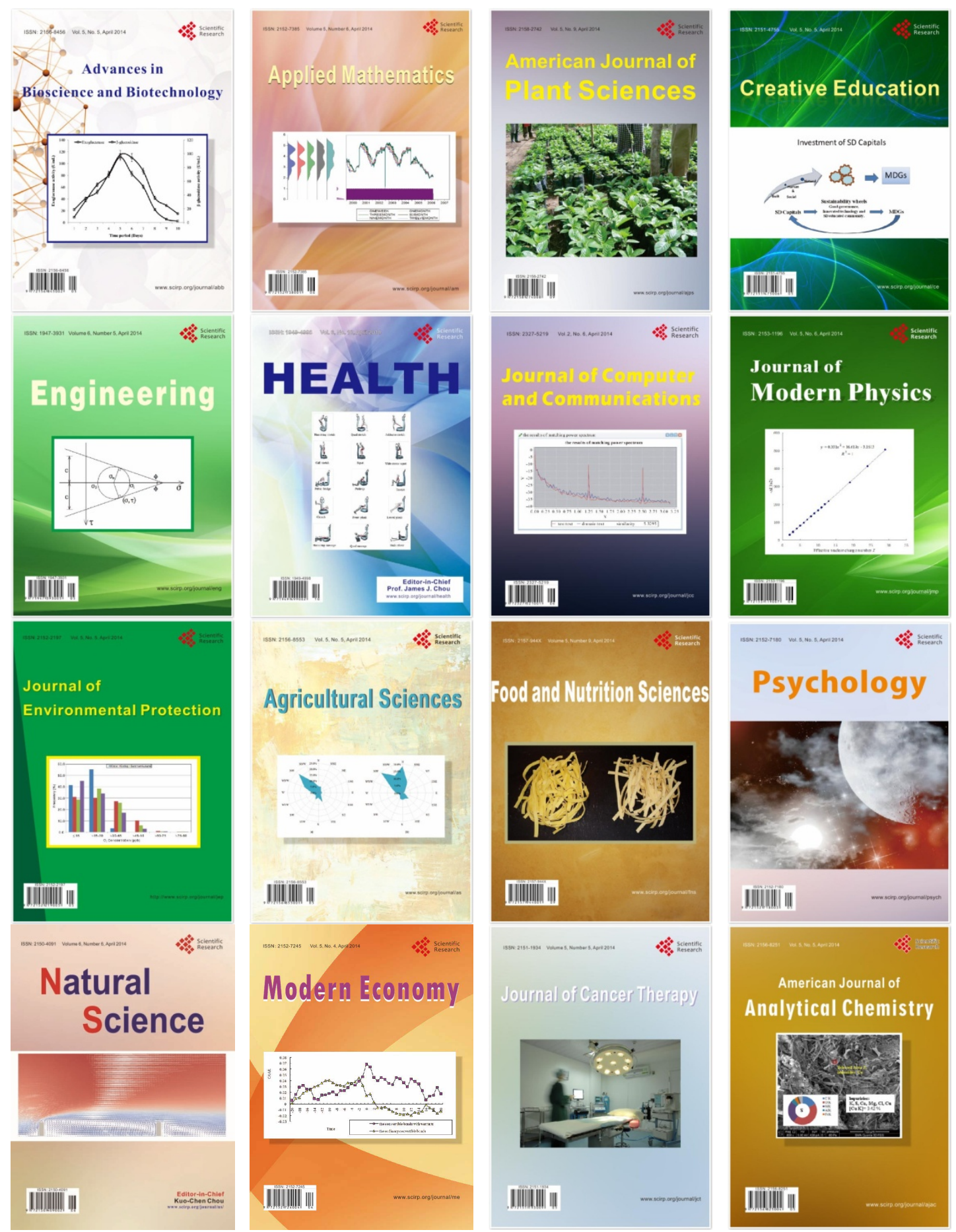